\title{
Equivalent forms of the Bessis-Moussa-Villani conjecture
}

\author{
Elliott H. Lieb* and Robert Seiringer ${ }^{\dagger}$ \\ Departments of Mathematics and Physics, Jadwin Hall, \\ Princeton University, P. O. Box 708, Princeton, New Jersey 08544
}

January 17, 2003

Dedicated with best wishes to Giovanni Jona-Lasinio on his $70^{\text {th }}$ birthday

\begin{abstract}
The BMV conjecture for traces, which states that $\operatorname{Tr} \exp (\mathrm{A}-\lambda \mathrm{B})$ is the Laplace transform of a positive measure, is shown to be equivalent to two other statements: (i) The polynomial $\lambda \mapsto \operatorname{Tr}(\mathrm{A}+\lambda \mathrm{B})^{p}$ has only non-negative coefficients for all $\mathrm{A}, \mathrm{B} \geq 0, p \in \mathbb{N}$ and (ii) $\lambda \mapsto \operatorname{Tr}(\mathrm{A}+$ $\lambda \mathrm{B})^{-p}$ is the Laplace transform of a positive measure for $\mathrm{A}, \mathrm{B} \geq 0$, $p>0$.
\end{abstract}

An intriguing conjecture [1], which is more than a quarter century old, was formulated by Bessis, Moussa and Villani (BMV) in an attempt to simplify the calculation of partition functions of quantum mechanical systems. It refers to a positivity property of traces of matrices which, if true, would permit the calculation of explicit error bounds in a sequence of approximations known as the Padé approximants.

The BMV conjecture is easy to state. Let A and B be any two Hermitian $n \times n$ matrices with $\mathrm{B} \geq 0$, and let $\lambda \in \mathbb{R}$. Then the function of $\lambda$ given by

$$
\lambda \mapsto \operatorname{Tr} \exp (\mathrm{A}-\lambda \mathrm{B})
$$

${ }^{*}$ Work partially supported by U.S. National Science Foundation grant PHY 0139984.

${ }^{\dagger}$ Erwin Schrödinger Fellow, supported by the Austrian Science Fund.

(c) 2003 by the authors. This paper may be reproduced, in its entirety, for non-commercial purposes. 
(with $\operatorname{Tr}=$ trace) is the Laplace transform of a positive measure on $[0, \infty)$.

Oddly, the positivity can be easily seen to be true for quantum mechanics defined by the Schrödinger equation for bosons without magnetic fields because the partition function in that case can be represented by Wiener integrals. This representation fails for fermions, and hence the importance of the conjecture for condensed matter physics.

The BMV conjecture appears naturally in other areas of matrix analysis as well and there is a significant literature devoted to it. For $2 \times 2$ matrices there is an easy proof of its correctness, but for $3 \times 3$ matrices neither a proof nor a counterexample is known to this day. Some recent work that shows the validity of the conjecture in some 'average' or 'typical' sense is in [3, 4. Ref. [7] contains a review.

The purpose of the present paper is to show that the BMV conjectured positivity is equivalent to two other positivity statements that appear to be somewhat different. One of them is, in fact, easier than the original conjecture because it involves only a statement about certain polynomials. Namely for every pair of positive matrices $A$ and $B$ and every positive integer $p$, the polynomial $\lambda \mapsto \operatorname{Tr}(\mathrm{A}+\lambda \mathrm{B})^{p}$ has all its coefficients positive. It is relatively easy to see that this statement implies the BMV conjecture but the striking fact, which is the main point of our paper, is that the BMV conjecture implies the polynomial statement. The current consensus is that the BMV conjecture is true, in which case our equivalence leads to a nonobvious and interesting theorem about traces. It also presents an alternative route to proving or disproving the BMV conjecture. We are indebted to D. Petz for urging us to write this note about the equivalences.

THEOREM 1. For fixed $n$ let $\mathrm{A}$ and $\mathrm{B}$ denote arbitrary hermitian $n \times n$ matrices over $\mathbb{C}$, and let $\lambda \in \mathbb{R}$. The following statements are equivalent:

(i) For all $\mathrm{A}$ and $\mathrm{B}$ positive, and all $p \in \mathbb{N}$, the polynomial $\lambda \mapsto \operatorname{Tr}(\mathrm{A}+\lambda \mathrm{B})^{p}$ has only non-negative coefficients.

(ii) For all $\mathrm{A}$ hermitian and $\mathrm{B}$ positive, $\lambda \mapsto \operatorname{Tr} \exp (\mathrm{A}-\lambda \mathrm{B})$ is the Laplace transform of a positive measure supported in $[0, \infty)$.

(iii) For all $\mathrm{A}$ positive definite and $\mathrm{B}$ positive, and all $p \geq 0, \lambda \mapsto \operatorname{Tr}(\mathrm{A}+$ $\lambda \mathrm{B})^{-p}$ is the Laplace transform of a positive measure supported in $[0, \infty)$.

Remark 1. A positive means that $\mathrm{A}$ is Hermitian and $(v, \mathrm{~A} v) \geq 0$ for all vectors $v$. Positive definite means positive and invertible. 
Remark 2. By Bernstein's theorem [2], a function of $\lambda$ defined for $\lambda \geq 0$ is the Laplace transform of a positive measure supported in $[0, \infty)$ if and only if it is completely monotone, i.e., its $r$-th derivative has the $\operatorname{sign}(-1)^{r}$ for all values of $\lambda \geq 0$.

Remark 3. In the case of $2 \times 2$ matrices, it is easy to see that statement (i) of Theorem 1 (and therefore all three statements) are true: there exists a basis in which both $A$ and $B$ have only non-negative entries, and therefore all coefficients of $\operatorname{Tr}(\mathrm{A}+\lambda \mathrm{B})^{p}$, which is independent of the choice of the basis, are necessarily non-negative. Some other special cases are treated in [6].

Remark 4. Note the logical structure in the proof that statement (iii) for $p \in \mathbb{N}$ implies item (i), which then implies (iii) for all $p>0$.

Remark 5. The proof will show (see (13)) that item (iii) is really a very simple corollary of item (ii). Indeed, the proof extends to the statement that $\lambda \mapsto \operatorname{Tr} f(\mathrm{~A}+\lambda \mathrm{B})$ is the Laplace transform of a positive measure supported in $[0, \infty)$ for $A \geq 0, B \geq 0$ whenever $f$ is the Laplace transform of a positive measure supported in $[0, \infty)$.

Remark 6. An important remark about item (i) is addressed in [5]. When $\operatorname{Tr}(\mathrm{A}+\lambda \mathrm{B})^{p}$ for $p \in \mathbb{N}$ is multiplied out, the coefficient of $\lambda^{r}$ is a sum of terms. [5] shows that some of these terms can be negative. The first interesting case occurs for $p=6$ and $r=3$. The term $\operatorname{Tr} \mathrm{A}^{2} \mathrm{~B}^{2} \mathrm{AB}$ can be negative even though $A$ and $B$ are positive. Nevertheless, the BMV conjecture implies that the sum of all terms of order $\lambda^{3}$ is non-negative. We cannot prove this, even for $n=3, r=3, p=6$.

The proofs of $(\mathrm{i}) \Rightarrow$ (ii) and (ii) $\Rightarrow$ (iii) will turn out to be very easy. The difficult direction is (iii) $\Rightarrow(\mathrm{i})$ and the following lemma is needed for this case.

LEMMA 1. Let a and $\mathrm{b}$ be hermitian $n \times n$ matrices over $\mathbb{C}$, with a positive definite. Define $\mathrm{A}=\mathrm{a}^{-1}$ and $\mathrm{B}=\mathrm{a}^{-1 / 2} \mathrm{ba}^{-1 / 2}$, and let $\lambda \in \mathbb{R}$. For all $p, r \in \mathbb{N}$

$$
\left.\frac{d^{r}}{d \lambda^{r}} \operatorname{Tr} \frac{1}{(\mathrm{a}+\lambda \mathrm{b})^{p}}\right|_{\lambda=0}=\left.\frac{p}{p+r}(-1)^{r} \frac{d^{r}}{d \lambda^{r}} \operatorname{Tr}(\mathrm{A}+\lambda \mathrm{B})^{p+r}\right|_{\lambda=0} .
$$

Proof. By induction it is easy to show that

$$
\frac{d^{r}}{d \lambda^{r}}(\mathrm{~A}+\lambda \mathrm{B})^{p+r}=r ! \sum_{\substack{0 \leq i_{1}, \ldots, i_{r+1} \leq p \\ \sum_{j} i_{j}=p}}(\mathrm{~A}+\lambda \mathrm{B})^{i_{1}} \mathrm{~B} \cdots \mathrm{B}(\mathrm{A}+\lambda \mathrm{B})^{i_{r+1}} .
$$


By taking the trace at $\lambda=0$ we obtain

$$
\left.I_{1} \equiv \frac{d^{r}}{d \lambda^{r}} \operatorname{Tr}(\mathrm{A}+\lambda \mathrm{B})^{p+r}\right|_{\lambda=0}=r ! \sum_{\substack{0 \leq i_{1}, \ldots, i_{r+1} \leq p \\ \sum_{j} i_{j}=p}} \operatorname{Tr} \mathrm{A}^{i_{1}} \mathrm{~B} \cdots \mathrm{BA}^{i_{r+1}} .
$$

Moreover, by similar arguments,

$$
\frac{d^{r}}{d \lambda^{r}} \frac{1}{(\mathrm{a}+\lambda \mathrm{b})^{p}}=(-1)^{r} r ! \sum_{\substack{1 \leq i_{1}, \ldots, i_{r+1} \leq p \\ \sum_{j}, i_{j}=p+r}} \frac{1}{(\mathrm{a}+\lambda \mathrm{b})^{i_{1}}} \mathrm{~b} \cdots \mathrm{b} \frac{1}{(\mathrm{a}+\lambda \mathrm{b})^{i_{r+1}}} .
$$

By taking the trace at $\lambda=0$ and using cyclicity, we get

$$
\left.I_{2} \equiv \frac{d^{r}}{d \lambda^{r}} \operatorname{Tr} \frac{1}{(\mathrm{a}+\lambda \mathrm{b})^{p}}\right|_{\lambda=0}=(-1)^{r} r ! \sum_{\substack{0 \leq i_{1}, \ldots, i_{r+1} \leq p-1 \\ \sum_{j} i_{j}=p-1}} \operatorname{Tr} \mathrm{AA}^{i_{1}} \mathrm{~B} \cdots \mathrm{BA}^{i_{r+1}}
$$

We have to show that

$$
I_{2}=\frac{p}{p+r}(-1)^{r} I_{1}
$$

To see this we rewrite $I_{1}$ in the following way. Define $p+r$ matrices $\mathrm{M}_{j}$ by

$$
\mathrm{M}_{j}=\left\{\begin{array}{l}
\mathrm{B} \quad \text { for } 1 \leq j \leq r \\
\mathrm{~A} \quad \text { for } r+1 \leq j \leq r+p
\end{array}\right.
$$

Let $\mathcal{S}_{n}$ denote the permutation group. Then

$$
I_{1}=\frac{1}{p !} \sum_{\pi \in \mathcal{S}_{p+r}} \operatorname{Tr} \prod_{j=1}^{p+r} \mathrm{M}_{\pi(j)}
$$

Because of the cyclicity of the trace we can always arrange the product such that $\mathrm{M}_{p+r}$ has the first position in the trace. Since there are $p+r$ possible locations for $\mathrm{M}_{p+r}$ to appear in the product above, and all products are equally weighted, we get

$$
I_{1}=\frac{p+r}{p !} \sum_{\pi \in \mathcal{S}_{p+r-1}} \operatorname{Tr} \mathrm{A} \prod_{j=1}^{p+r-1} \mathrm{M}_{\pi(j)}
$$


On the other hand,

$$
I_{2}=(-1)^{r} \frac{1}{(p-1) !} \sum_{\pi \in \mathcal{S}_{p+r-1}} \operatorname{Tr} \mathrm{A} \prod_{j=1}^{p+r-1} \mathrm{M}_{\pi(j)}
$$

so we arrive at the desired equality.

Proof of Theorem 1. (i) $\Rightarrow$ (ii): By continuity of the trace,

$$
\operatorname{Tr} \exp (\mathrm{A}-\lambda \mathrm{B})=e^{-\|\mathrm{A}\|} \sum_{k=0}^{\infty} \frac{1}{k !} \operatorname{Tr}(\mathrm{A}+\|\mathrm{A}\| \mathbb{I}-\lambda \mathrm{B})^{k}
$$

where $\mathbb{I}$ denotes the identity matrix. It follows from Bernstein's theorem and (i) that the right side is the Laplace transform of a positive measure supported in $[0, \infty)$.

(ii) $\Rightarrow$ (iii): This follows from taking the trace of the matrix equation

$$
\frac{1}{(\mathrm{~A}+\lambda \mathrm{B})^{p}}=\frac{1}{\Gamma(p)} \int_{0}^{\infty} \exp [-t(\mathrm{~A}+\lambda \mathrm{B})] t^{p-1} d t
$$

(iii) $\Rightarrow($ i): It suffices to assume (iii) only for $p \in \mathbb{N}$. For invertible $A$ we observe that the $r$-th derivative of $\operatorname{Tr}(\mathrm{a}+\lambda \mathrm{b})^{-p}$ at $\lambda=0$ is related to the coefficient of $\lambda^{r}$ in $\operatorname{Tr}(\mathrm{A}+\lambda \mathrm{B})^{p}$ as given by (2) with $\mathrm{A}, \mathrm{a}, \mathrm{B}, \mathrm{b}$ related as in Lemma 1. The left side of (2) has the sign $(-1)^{r}$ because it is the derivative of a function that is the Laplace transform of a positive measure supported in $[0, \infty)$. Thus the right side has the correct sign as stated in item (i). The case of non-invertible $A$ follows from continuity.

\section{References}

[1] D. Bessis, P. Moussa and M. Villani, Monotonic converging variational approximations to the functional integrals in quantum statistical mechanics, J. Math. Phys. 16, 2318-2325 (1975).

[2] W. F. Donoghue, Monotone matrix functions and analytic continuation, Springer (1974).

[3] M. Fannes and D. Petz, On the function $\operatorname{Tr} e^{H+i t K}$, Int. J. Math. and Math. Sci. 29, 389-394 (2001). 
[4] M. Fannes and D. Petz, Perturbation of Wigner matrices and a conjecture, preprint.

[5] C. R. Johnson and C. J. Hillar, Eigenvalues of words in two positive definite letters, SIAM J. Matrix Anal. Appl. 23, 916-928 (2002).

[6] M. L. Mehta and K. Kumar, On an integral representation of the function $\operatorname{Tr}[\exp (\mathrm{A}-\lambda \mathrm{B})]$, J. Phys. A 9, 197-206 (1976).

[7] P. Moussa, On the representation of $\operatorname{Tr}\left(e^{(\mathrm{A}-\lambda \mathrm{B})}\right)$ as a Laplace Transform, Rev. Math. Phys. 12, 621-655 (2000). 